\title{
Extreme anorexia nervosa: medical findings, outcomes, and inferences from a retrospective cohort
}

\author{
Dennis Gibson ${ }^{1,2}$, Ashlie Watters ${ }^{1,2}$, Jeana Cost ${ }^{1,3}$, Margherita Mascolo ${ }^{2}$ and Philip S. Mehler ${ }^{1,2,3^{*}}$
}

\begin{abstract}
Background: Extreme anorexia nervosa (AN) is defined as a BMl $<15 \mathrm{~kg} / \mathrm{m}^{2}$ in those meeting DSM-V diagnostic criteria for AN. This study seeks to define the frequency of medical complications in this group of patients in order to help inform the care of individuals $<65 \%$ ideal body weight who seek treatment for their extreme eating disorders.

Methods: Through retrospective chart review and computerized data collection, we obtained the baseline characteristics and medical findings of 281 adult patients, with AN restricting and binge-purge subtypes, admitted to the ACUTE unit for medical stabilization between May 2013 and August 2018.

Results: In this population, with a mean admitting BMI of $12.1 \mathrm{~kg} / \mathrm{m}^{2}$ (range $=7.5-15.7$ ), $56 \%$ admitted with bradycardia, 45\% demonstrated increased liver function tests (LFTs) on admission, 64\% admitted with leukopenia, $47 \%$ with anemia, and 20\% presented with thrombocytopenia. During admission, 38\% developed hypoglycemia, $35 \%$ developed refeeding hypophosphatemia, nearly $33 \%$ of patients developed edema, and low bone mineral density was diagnosed in almost $90 \%$ of the patients. Highly elevated LFTs ( $>3 x$ upper limits of normal) predicted hypoglycemia, and low BMI predicted refeeding hypophosphatemia $(p=.001)$.
\end{abstract}

Conclusions: Although conclusions drawn from the findings presented in this descriptive study must be tempered by relevant clinical judgement, these findings showcase that patients with extreme AN are at significantly increased risk for many serious medical complications secondary to their state of malnutrition and also with initial refeeding.

Keywords: Extreme anorexia nervosa, Medical complications, Malnutrition, Outcomes, Refeeding

\section{Plain English summary}

Extreme anorexia nervosa (AN) is defined as a $\mathrm{BMI}<15$ $\mathrm{kg} / \mathrm{m}^{2}$ in those meeting DSM-5 diagnostic criteria for AN. However, the frequency of medical complications specific to those meeting this greatest amount of weight loss is unknown. This retrospective study seeks to better define the frequency of medical complications in

\footnotetext{
* Correspondence: Philip.mehler@dhha.org

'ACUTE Center for Eating Disorders at Denver Health, 723 Delaware St, Pav M 3rd floor, Denver, CO 80204, USA

${ }^{2}$ Department of Medicine, University of Colorado School of Medicine, 13001 E 17th PI, Aurora, CO 80045, USA

Full list of author information is available at the end of the article
}

individuals $<65 \%$ ideal body weight admitting to an inpatient medical stabilization unit. We found that patients with extreme AN present with similar medical complications, although at a more frequent occurrence, to those at higher percent ideal body weights. Low bone mineral density is an almost universal finding in this population, with $45 \%$ of individuals presenting with osteopenia and $43 \%$ with osteoporosis. $35 \%$ of individuals developed hypophosphatemia and 38\% developed hypoglycemia in this population, both more frequent in the restricting than binge-purge subtype of AN. Elevated liver function tests predict the development of

(c) The Author(s). 2020 Open Access This article is licensed under a Creative Commons Attribution 4.0 International License, which permits use, sharing, adaptation, distribution and reproduction in any medium or format, as long as you give appropriate credit to the original author(s) and the source, provide a link to the Creative Commons licence, and indicate if changes were made. The images or other third party material in this article are included in the article's Creative Commons licence, unless indicated otherwise in a credit line to the material. If material is not included in the article's Creative Commons licence and your intended use is not permitted by statutory regulation or exceeds the permitted use, you will need to obtain permission directly from the copyright holder. To view a copy of this licence, visit http://creativecommons.org/licenses/by/4.0/ The Creative Commons Public Domain Dedication waiver (http://creativecommons.org/publicdomain/zero/1.0/) applies to the data made available in this article, unless otherwise stated in a credit line to the data. 
hypoglycemia. Patients with extreme AN are at a significantly increased risk for many serious medical complications secondary to their state of malnutrition and also with initial refeeding.

\section{Background}

Anorexia nervosa (AN) is a serious psychiatric illness that leads to medical complications which can adversely involve every organ system [1]. Mortality remains one of the highest of any psychiatric disorders [2] with cause of death often attributed to the medical complications of malnutrition [3, 4]. The prognosis for AN is somewhat guarded, with less than one-third of individuals recovered at 9 years and approximately two-thirds of individuals recovered when followed up to 22 years [5]. Correlational data suggest that full weight restoration is a predictor of a more favorable outcome at follow up [6, 7], but the lower the percentage of ideal body weight (\%IBW) at presentation, the worse the prognosis due to more medical complications [8]. These patients may often undergo unnecessary evaluations for their medical complaints and languish in medical hospitals without definitive care.

The ACUTE Center for Eating Disorders at Denver Health (ACUTE), established in 2004, is a telemetry medical stabilization unit, with national and international draw, that singularly specializes in the care of the most medically compromised patients with eating disorders, as the typical patient admitted to ACUTE meets criteria for an extreme form of the illness per the DSM-V severity index for eating disorders [9]. In this article we present data from a series of 281 patients admitted to ACUTE, as the first study of a comprehensive evaluation of medical complications observed in patients with extreme AN.

\section{Methods}

Patients admitted to ACUTE are regularly transferred by air ambulance from medical hospitals where they had been admitted with various serious medical conditions such as syncope, cardiac arrhythmias, hypoglycemic seizures, failure to thrive, severe electrolyte imbalances and liver failure. Typical ACUTE patients have AN, restricting subtype (AN-R) or binge-purge subtype (AN-BP), with body weights less than $70 \%$ of ideal body weight (IBW), and with comorbid medical complications and marked physical deconditioning. Percent IBW is calculated using the Hamwi method [10]. Multidisciplinary expertise, in the medical complications of AN and refeeding, is exclusively provided by embedded full-time internal medicine physicians, psychiatrists, registered dietitians, clinical psychologists, physical therapists, occupational therapists, social workers, nurses, and certified nursing assistants. Standard blood tests, anthropometrics, 12-lead electrocardiograms (EKG), and vital signs with orthostatic blood pressures are obtained for all patients upon admission. Laboratory values, such as glucose, phosphorus, and liver function tests (LFTs) are closely monitored until stabilized. Daily blinded weights were also obtained at approximately the same time daily for each patient while wearing only a gown. The multidisciplinary team then facilitates the initiation of aggressive, albeit judicious, nutritional rehabilitation. Progressive oral (PO) kcals, increased until meeting our unit's expected weight trends of $0.2 \mathrm{~kg} /$ day, is provided with conversion to enteral nutrition (EN) only when patients are unable to tolerate $\mathrm{PO}$ intake; rarely, peripheral parenteral nutrition/total parental nutrition (PPN/TPN) is provided when unable to tolerate enteral feeds for significant physiologic reasons. In addition, all meal completion is monitored by a care patient safety aide with training in eating disorders. Once the patient has met ACUTE's discharge criteria, as well as meeting the accepting treatment program's admission criteria, they are discharged.

This retrospective study design involves 281 male and female adult patients who weighed less than $65 \%$ of IBW and admitted to ACUTE for medical stabilization, between May 18, 2013 and August 30, 2018. It compares baseline characteristics and medical complications between restricting and binge-purge subtypes. Patients were excluded if they were hospitalized for less than 72 $\mathrm{h}$ or if their percent IBW was above $65 \%$ on admission.

Patient demographics, anthropometric measurements, dietary intake, bone mineral density (BMD) tests, and EKG data were obtained from manual chart review. Nursing assessments and laboratory testing results were queried from the hospital database. Duration of illness and mode and frequency of purging behaviors were selfreported and recorded on admission. Hypothermia was defined as body temperature less than $36^{\circ} \mathrm{C}$ and bradycardia was defined as heart rate less than 60 beats per minute (bpm). Severe hypoglycemia was defined as blood glucose $<2.2 \mathrm{mmol} / \mathrm{L}$. Hypophosphatemia was defined as a serum value less than $0.87 \mathrm{mmol} / \mathrm{L}$. Patients with hypophosphatemia on admission were excluded from the risk factor analysis for refeeding hypophosphatemia. Dual-energy X-ray absorptiometry (DXA) scans and lipodystrophy analysis/whole body scans were performed using the Hologic QDR Series model DiscoveryW if not already completed within the previous 2 years. BMD was assessed using the lowest regional (hip, femoral neck, and lumbar spine) Z-score or T-score (if patient was 50 years old or older). On ACUTE, osteoporosis is diagnosed if the $\mathrm{Z}$ or $\mathrm{T}$-score is less than -2.5 and osteopenia is diagnosed when the lowest $\mathrm{Z}$ or $\mathrm{T}$ score is between -1 and -2.5 . Nadir body mass index (BMI) and percent IBW are listed to reflect the lowest 
body weight experienced during treatment. Admission and discharge caloric intake were based on those amounts consumed on the first and last day the patient was on the unit for $24 \mathrm{~h}$. The study was reviewed and approved by the Colorado Multiple Institutional Review Board.

\section{Statistical analysis}

Univariate statistics were used to describe the cohort. Shapiro-Wilks test was used to determine the distribution of continuous variables. Depending on the distribution, patient characteristics, used to describe the medical complications on the ACUTE unit, were ascertained by Mann-Whitney $U$ procedures, Pearson chi-square tests, and independent sample $t$-tests. When appropriate, degrees of freedom are shown in parentheses after the test statistic. Logistic regression analyses were used to investigate associations between medical complications with clinical or biochemical variables. Two-tailed $p$ values $<$ 0.05 were considered statistically significant, and all analyses were completed using SAS Enterprise Guide software version 7.1 (SAS Institute, Cary, NC).

\section{Results}

\section{Baseline characteristics}

Demographic characteristics and statistical comparisons between AN subtypes, in regard to percent IBW, BMI, and caloric intake, are listed in Table 1. A large majority of ACUTE patients (91\%) were referrals from outside of Colorado. Most of the 281 study patients were female (91\%), 62\% were diagnosed as AN-R subtype, and the age range of the patients was 18-66 years with a median age of 28 years. The average BMI on admission was 12.1 $\mathrm{kg} / \mathrm{m}^{2}(\mathrm{SD}=1.3$; range $=7.5-15.7)$ and average percent IBW was $57.7 \%(\mathrm{SD}=5.5$; range $=34.8-65.0)$. The median duration of illness was 10 years $(\mathrm{IQR}=5-20)$. For females, duration of illness was significantly higher with a median of 11 years (IQR $=6-21$ ) and for males, the median was 4 years (IQR $=1-10), p<.0001$. Out of the total cohort, $82(29 \%)$ patients reported vomiting as a method of purging, 41 (15\%) reported using laxatives, and $7(2 \%)$ reported using diuretics. Many of these patients used just one method of purging, but some used two or even all three of the methods. However, many patients were unable to recall the frequency of their purging behaviors.

\section{Patient care and discharge}

Patients hospitalized at ACUTE had a median length of stay of 22 days (IQR $=15-30)$. The average percent IBW increased by $12 \%(\mathrm{SD}=8.4)$, and average $\mathrm{BMI}$ increased by $2.5 \mathrm{~kg} / \mathrm{m}^{2}(\mathrm{SD}=1.5)$ by time of discharge (Table 1$)$. Patients with AN-BP gained 0.9 more kilograms per week compared to patients with AN-R $(p<.0001)$.
About one third of the patients developed edema during their hospital stay and half of those patients, almost entirely patients with AN-BP, were prescribed spironolactone to treat their excessive weight trends and/or edema. Patients with AN-BP, who were prescribed spironolactone, gained $0.7 \mathrm{~kg}$ more per week than patients with AN-BP who were not prescribed spironolactone.

Most of the patients discharged voluntarily $(n=209)$ with an average percent IBW of $69.4 \%(\mathrm{SD}=7.6)$; however, 37 patients discharged from ACUTE on a mental health hold (MHH), 21 discharged on a short-term certification (STC), and 14 discharged against medical advice (AMA).

All patients were medically stabilized prior to discharge. Two hundred forty-one patients (86\%) discharged to an inpatient or residential program; however, 16 patients (6\%) discharged to a partial hospitalization program (PHP), an outpatient team, or to another form of care, and the remaining 24 patients (9\%) were discharged home due to mitigating circumstances. No patient died on the ACUTE unit during their medical stabilization and only 20 patients (7\%) ever required brief transfer to the Denver Health medical intensive care unit (ICU) for conditions such as critical hyponatremia $(<116 \mathrm{mmol} / \mathrm{L})$, critical hypokalemia (< $1.8 \mathrm{mmol} / \mathrm{L}$ ), refractory hypoglycemia requiring intravenous (IV) glucose and frequent point of care glucose monitoring, small bowel obstructions requiring surgery, cardiogenic shock, upper gastrointestinal bleed requiring transfusion, and two with sustained ventricular tachycardia. Forty-three patients (15\%) were deemed high fall risk upon admission, and 15 patients (5\%) were initially incapable of ambulation. All received intensive physical therapy and were able to ambulate on their own at the time of discharge.

\section{Nutrition}

On admission, initial caloric intake for females ranged from 800 to $2450 \mathrm{kcals}$ with an average of $1431 \mathrm{kcal} /$ day $(\mathrm{SD}=191)$ and initial caloric intake for males was significantly higher at $1714 \mathrm{kcal} /$ day $(\mathrm{SD}=507)(p=.01)$. The vast majority of patients were refed via progressive oral nutrition. There were 50 patients $(18 \%)$ who required enteral feeds; 18 patients (6\%) required some parenteral nutrition. Average daily caloric intake at the time of discharge for females was $3119 \mathrm{kcal} /$ day $(\mathrm{SD}=733)$ and $3744 \mathrm{kcal} /$ day for males $(\mathrm{SD}=829)$. During their ACUTE stay, patients gained $2 \mathrm{~kg} / \mathrm{wk}$. $(\mathrm{SD}=1.5)$. There was not a significant difference in weight gain per week between genders $(p=.84)$.

\section{Medical findings}

Patients were hypothermic during 35\% of their total admission time at ACUTE with low percent IBW as a predictor of hypothermia $(p<.0001)$. For patients with 
Table 1 Characteristics of study participants

\begin{tabular}{|c|c|c|c|c|c|c|c|c|}
\hline \multirow[b]{2}{*}{ Patient Characteristics } & \multicolumn{2}{|c|}{ Cohort $(\boldsymbol{N}=281)$} & \multicolumn{2}{|c|}{ AN-R $(\boldsymbol{N}=175)$} & \multicolumn{2}{|c|}{ AN-BP $(\boldsymbol{N}=106)$} & & \\
\hline & $\mathrm{N}$ & $\%$ & $\mathrm{~N}$ & $\%$ & $\mathrm{~N}$ & $\%$ & & \\
\hline Female & 256 & 91 & 158 & 62 & 98 & 38 & & \\
\hline Male & 25 & 9 & 17 & 68 & 8 & 32 & & \\
\hline \multicolumn{9}{|l|}{ Admitted from } \\
\hline Home & 219 & 78 & 128 & 58 & 91 & 42 & & \\
\hline Hospital transfer & 37 & 13 & 29 & 78 & 8 & 22 & & \\
\hline Inpatient eating disorder program & 23 & 8 & 17 & 74 & 6 & 26 & & \\
\hline Other & 2 & $<1$ & 1 & 50 & 1 & 50 & & \\
\hline \multirow[t]{2}{*}{ Referral from outside Colorado } & 257 & 91 & 161 & 63 & 96 & 37 & & \\
\hline & Mean $^{\mathrm{a}}$ & $S D^{a}$ & Mean $^{a}$ & $S D^{a}$ & Mean $^{a}$ & $S D^{a}$ & $t$-test ${ }^{b}$ & $p$ value \\
\hline Age & 28 & $22-37$ & 27 & $22-38$ & 31 & $22-37$ & 15,900 & 0.15 \\
\hline Length of hospitalization (days) & 22 & $15-30$ & 23 & $16-33$ & 21 & $13-28$ & 13,754 & 0.07 \\
\hline \multicolumn{9}{|l|}{ Percent of IBW } \\
\hline Admission & 57.7 & 5.5 & 57.2 & 5.5 & 58.4 & 5.4 & 1.75 & 0.08 \\
\hline Nadir & 57 & 6.2 & 56.5 & 6.1 & 57.9 & 6.5 & 1.81 & 0.07 \\
\hline Discharge & 69 & 7.4 & 67.7 & 6.2 & 71.2 & 8.7 & 3.61 & $<.001$ \\
\hline Change in \%lBW & 12 & 8.4 & 11.2 & 7.5 & 13.3 & 9.6 & 2.03 & 0.04 \\
\hline \multicolumn{9}{|l|}{ BMI $\left(\mathrm{kg} / \mathrm{m}^{2}\right)$} \\
\hline Admission & 12.1 & 1.3 & 12 & 1.3 & 12.2 & 1.3 & 1.01 & 0.31 \\
\hline Nadir & 12 & 1.4 & 11.9 & 1.4 & 12.1 & 1.6 & 0.91 & 0.37 \\
\hline Discharge & 14.5 & 1.5 & 14.2 & 1.3 & 14.9 & 1.7 & 3.5 & $<.001$ \\
\hline Change in BMl & 2.5 & 1.5 & 2.3 & 1.4 & 2.8 & 1.7 & 2.8 & $<.001$ \\
\hline Weekly weight gain (kg) & 2 & 1.5 & 1.7 & 0.9 & 2.6 & 2.0 & 4.26 & $<.0001$ \\
\hline \multicolumn{9}{|l|}{ Daily caloric intake (kcal) } \\
\hline Admission & 1457 & 249 & 1477 & 269 & 1424 & 209 & -1.74 & 0.06 \\
\hline Discharge & 3175 & 762 & 3282 & 763 & 2996 & 728 & -3.1 & 0.002 \\
\hline
\end{tabular}

$A N-R$ Anorexia nervosa restricting subtype, $A N-B P$ Anorexia nervosa binge-purge subtype, $I B W$ ldeal body weight, $B M I$ Body mass index ${ }^{a}$ Median and IQR for not normally distributed variables

${ }^{\mathrm{b}}$ Mann-Whitney $U$ test

AN-R, the average percent IBW on the last hospital day of hypothermia was $61.5 \%(\mathrm{SD}=6.3)$ (Table 2).

There were 158 patients (56\%) who were bradycardic on arrival to ACUTE, with the lowest recorded heart rate being $26 \mathrm{bpm}$, and the majority of those patients were diagnosed with AN-R. Admission BMI $(r=.85$, $p<.0001)$ and percent IBW $(r=.85, p<.0001)$ were highly correlated with bradycardia. For the patients with bradycardia, their heart rate had somewhat improved by median day $4(\mathrm{IQR}=1-9)$. Findings from the admission EKG revealed that the average QTc was normal at 427 ms $(\mathrm{SD}=34.4)$. Patients with AN-BP had significantly longer average admission QTc $(436 \mathrm{~ms}, \mathrm{SD}=37.2)$ than patients with AN-R (421 ms, SD = 31.4), $(p<.001)$.

Cardiac axis was mostly normal. There were 58 patients with right axis deviation but only 10 patients presented with left axis deviation. Five patients presented with a junctional rhythm. Admission BMI was not a significant predictor of either right axis deviation $(p=.45)$ or of developing a junctional rhythm $(p=.15)$.

Just over half of the patients admitted with low serum prealbumin values $(<0.2 \mathrm{~g} / \mathrm{L})$. Low serum prealbumin positively correlated with admission percent IBW $(r=$ $0.6, p<.0001)$. Admission serum albumin and alkaline phosphatase levels were within normal ranges. However, 127 (45\%) patients had elevated admission aspartate aminotransferase (AST) values (above $40 \mathrm{U} / \mathrm{L}$ ) and 145 (52\%) patients had elevated alanine aminotransferase (ALT) values (above $45 \mathrm{U} / \mathrm{L}$ ). Patients with AN-R had significantly higher AST $(p<.0001)$ and $\operatorname{ALT}(p<.0001)$ values compared to patients with AN-BP (Table 3).

The incidence of increased liver function tests (LFTs), on admission, is correlated with low BMI $\left[X^{2}(1)=13.3\right.$, $p<.005]$. Of the 281 study patients, only $85(30 \%)$ had normal LFTs (AST $<41 \mathrm{U} / \mathrm{L}$ and ALT $<46 \mathrm{U} / \mathrm{L}$ ) throughout their hospitalization. One hundred one (36\%) had 
Table 2 Medical complications between AN-R and AN-BP subtypes

\begin{tabular}{|c|c|c|c|c|c|c|c|c|c|c|c|c|}
\hline & \multicolumn{2}{|c|}{ Cohort $(\boldsymbol{N}=281)$} & \multicolumn{4}{|c|}{$\begin{array}{l}\text { AN-R } \\
(\boldsymbol{N}=175)\end{array}$} & \multicolumn{4}{|c|}{$\begin{array}{l}\text { AN-BP } \\
(\boldsymbol{N}=106) \\
\end{array}$} & \multirow[b]{2}{*}{$t$-test ${ }^{b}$} & \multirow[b]{2}{*}{$\boldsymbol{p}$ value } \\
\hline & $\overline{\mathrm{N}}$ & $\%$ & $\bar{N}$ & $\%$ & Mean $^{\mathbf{a}}$ & $S D^{a}$ & $\bar{N}$ & $\%$ & Mean $^{a}$ & $S D^{a}$ & & \\
\hline \multicolumn{13}{|l|}{ Body Temperature } \\
\hline Hypothermic during stay $\left(<36^{\circ} \mathrm{C}\right)$ & 168 & 60 & 110 & 65 & 35.8 & 0.1 & 58 & 35 & 35.8 & 0.2 & 1.59 & 0.11 \\
\hline Last hospital day $<36^{\circ} \mathrm{C}$ & & & & & 10 & $4-19$ & & & 11 & $5-21$ & 6084 & 0.85 \\
\hline$\%$ IBW on last day $<36^{\circ} \mathrm{C}$ & & & & & 61.5 & 6.3 & & & 65.8 & 7.2 & 4.12 & $<.0001$ \\
\hline \multicolumn{13}{|l|}{ Heart Rate and EKG on Admission } \\
\hline Heart rate (bpm) & & & & & 55.3 & 13.1 & & & 60.7 & 12.5 & 3.37 & 0.0009 \\
\hline Nadir heart rate (bpm) during stay & & & & & 50.1 & 11.8 & & & 54.3 & 3.9 & 3.06 & 0.002 \\
\hline QTc value (ms) & & & & & 421 & 31.4 & & & 436 & 37.2 & 1.41 & $<.001$ \\
\hline High QTc (480-500 ms) & 7 & 3 & 2 & 29 & & & 5 & 71 & & & & \\
\hline Critically high QTc (> 500 ms) & 7 & 3 & 3 & 43 & & & 4 & 57 & & & & \\
\hline Junctional rhythm & 5 & 2 & 1 & 20 & & & 4 & 80 & & & & \\
\hline Right axis & 58 & 21 & 40 & 69 & & & 18 & 31 & & & & \\
\hline \multicolumn{13}{|l|}{ Liver Function } \\
\hline All normal LFTs (AST < 41; ALT < 46 U/L) & 85 & 30 & 36 & 42 & & & 49 & 58 & & & & \\
\hline Modestly Abnormal (up to $3 x$ normal) & 101 & 36 & 60 & 59 & & & 41 & 41 & & & & \\
\hline Severely abnormal (> 3x normal) & 95 & 34 & 79 & 83 & & & 16 & 17 & & & & \\
\hline Highest AST & & & & & 197 & $145-348$ & & & 238 & $136-537$ & 383 & 0.76 \\
\hline Highest ALT & & & & & 270 & $197-485$ & & & 261 & $196-518$ & 618 & 0.65 \\
\hline Hospital day LFTs peaked & & & & & 4 & $0-12$ & & & 5 & $0-12$ & 6381 & 0.21 \\
\hline \multicolumn{13}{|l|}{ Blood Glucose } \\
\hline Hypoglycemia (< $3.3 \mathrm{mmol} / \mathrm{L})$ & 107 & 38 & 77 & 72 & 3.0 & $2.6-3.2$ & 30 & 28 & 2.9 & $2.8-3.1$ & 1637 & 0.45 \\
\hline Last hospital day glucose $<3.3 \mathrm{mmol} / \mathrm{L}$ & & & & & 2 & $1-2$ & & & 1 & $0-2$ & 1963 & 0.06 \\
\hline Severe hypoglycemia $(<2.2 \mathrm{mmol} / \mathrm{L})^{c}$ & 24 & 9 & 20 & 83 & 1.8 & $1.6-2.0$ & 4 & 17 & 1.9 & $1.4-2.1$ & & \\
\hline Last hospital day glucose $<2.2 \mathrm{mmol} / \mathrm{L}$ & & & & & 1 & $0-3$ & & & 1 & $0-3$ & & \\
\hline
\end{tabular}

$A N-R$ Anorexia nervosa restricting subtype, $A N-B P$ Anorexia nervosa binge-purge subtype, IBW Ideal body weight, $E K G$ Electrocardiogram, $b p m$ Beats per minute, LFT Liver function tests, AST Aspartate aminotransferase test, ALT Alanine aminotransferase test

aedian (IQR) for not normally distributed variables

${ }^{\mathrm{b}}$ Mann-Whitney $U$ test

'There were too few values for an analysis between AN subtypes and severe hypoglycemia

modestly elevated LFTs and 95 patients (34\%) had LFTs of greater than three times normal during their hospitalization (Table 2). Modestly abnormal LFTs predicted thrombocytopenia (serum platelet count $<15010^{9}$ $\left.\mathrm{L}^{-1}\right)$ on admission $(p=.002)$ but did not predict hypoglycemia $(p=0.43)$. However, highly elevated LFTs $(>3 \mathrm{X}$ normal) did predict the presence of hypoglycemia $(p=.005)$.

More than one third (38\%) of the patients experienced hypoglycemia while on ACUTE. Of these, 24 (9\%) admitted with severe hypoglycemia $(<2.2 \mathrm{mmol} /$ L.). Of the total 281 patients, 77 patients $(27 \%)$ with AN-R developed hypoglycemia and 20 patients developed severe hypoglycemia. There were only 30 patients (11\%) with AN-BP who developed hypoglycemia and only four developed severe hypoglycemia (Table 2).
Both admission sodium and potassium values, were lower in patients with AN-BP compared to patients with AN-R $(M=135 \mathrm{mmol} / \mathrm{L}, \mathrm{SD}=6.4$ vs. $\mathrm{M}=137.6 \mathrm{mmol} / \mathrm{L}$, $\mathrm{SD}=4.5, p<.0001)$. Hypokalemia was most commonly treated with a combination of oral potassium and IV fluids (47\%), only oral potassium (28\%), oral and IV potassium along with IV fluids (13\%), combination oral and IV potassium (9\%), or only IV fluids (3\%). Only eight patients of those developing hypokalemia $(n=93)$ required transfer to the ICU for critically low values. Hyponatremia was most commonly treated with slow IV saline (50\%), 34\% were allowed to auto-correct without any particular intervention aside from nutrition and fluid intake, and $13 \%$ required fluid restriction due to a diagnosis of syndrome of inappropriate antidiuretic hormone (SIADH). Four (5\%) of the total 82 patients, who developed hyponatremia, required transfer to the ICU for treatment of a critical value. 
Table 3 Admission laboratory values between AN subtypes $(N=281)^{a}$

\begin{tabular}{|c|c|c|c|c|c|c|}
\hline \multirow[b]{2}{*}{ Laboratory Values (reference range) } & \multicolumn{2}{|l|}{ AN-R } & \multicolumn{2}{|l|}{ AN-BP } & \multirow[t]{2}{*}{$\boldsymbol{t}$-test ${ }^{\mathbf{b}}$} & \multirow[t]{2}{*}{$\boldsymbol{p}$ value } \\
\hline & Mean $^{c}$ & $S^{c}$ & Mean $^{c}$ & $S D^{c}$ & & \\
\hline Sodium (135-143 mmol/L) & 137.6 & 4.5 & 135 & 6.4 & -4.01 & $<.0001$ \\
\hline Potassium (3.6-5.1 mmol/L) & 3.8 & 0.4 & 3.4 & 0.7 & -6.39 & $<.0001$ \\
\hline Carbon Dioxide (18-27 mmol/L) & 27.7 & 3.3 & 29.3 & 7.3 & 2.57 & 0.01 \\
\hline Glucose (3.3-11.0 mmol/L) & 4.57 & $3.91-5.89$ & 5.26 & $4.29-6.0$ & 16,507 & 0.009 \\
\hline Calcium (2.02-2.6 mmol/L) & 2.1 & 0.1 & 2.2 & 0.2 & 5.11 & $<.001$ \\
\hline Phosphorus (0.87-1.58 mmol/L) & 1.03 & 0.2 & 1.1 & 0.4 & 1.69 & 0.09 \\
\hline Magnesium (0.65-1.1 mmol/L) & 0.95 & 0.1 & 0.98 & 0.2 & 1.60 & 0.11 \\
\hline BUN $(0.21-0.79 \mathrm{mmol} / \mathrm{L})$ & 0.57 & $0.36-0.86$ & 0.50 & $0.32-0.75$ & 13,987 & 0.07 \\
\hline Creatinine $(44.2-122.9 \mu \mathrm{mol} / \mathrm{L})$ & 59.3 & 19.1 & 73.9 & 28.6 & 4.65 & $<.0001$ \\
\hline AST (10-40 U/L) & 49 & $26-113$ & 27 & $21-42$ & 11,730 & $<.0001$ \\
\hline $\operatorname{ALT}(7-45 \mathrm{U} / \mathrm{L})$ & 73 & $37-194$ & 32 & $24-50$ & 10,671 & $<.0001$ \\
\hline Total bilirubin $(17.1-20.5 \mu \mathrm{mol} / \mathrm{L})$ & 8.6 & $6.8-12.0$ & 6.8 & $5.1-15.4$ & 14,294 & 0.16 \\
\hline Albumin (30-53 g/L) & 38.5 & 4.9 & 37.9 & 5.9 & -0.85 & 0.39 \\
\hline Alkaline phosphatase (35-137 U/L) & 67 & $51-97$ & 68 & $54-95$ & 15,213 & 0.68 \\
\hline Total protein (60-82 g/L) & 67.0 & 7.5 & 68.7 & 9.1 & 1.78 & 0.08 \\
\hline WBC $\left(4.5-10.010^{9} \mathrm{~L}^{-1}\right)$ & 3.8 & 1.6 & 5.5 & 3.3 & 5.91 & $<.0001$ \\
\hline Neutrophils (\%) (48.0-69.0\%) & 56.9 & 12.6 & 57.4 & 14.5 & 0.27 & 0.79 \\
\hline Lymphocytes (\%) (21.0-43.0\%) & 34.4 & 12.4 & 33.3 & 13.5 & -0.71 & 0.48 \\
\hline Platelet count $\left(150-40010^{9} \mathrm{~L}^{-1}\right)$ & 192 & $142-250$ & 271 & $201-387$ & 19,308 & $<.0001$ \\
\hline Hematocrit (\%) (37.0-47.0\%) & 36.6 & 5.8 & 37.6 & 5.6 & 1.4 & 0.16 \\
\hline MCV (80-100 fL) & 94.6 & 6.7 & 91.1 & 6.7 & -4.27 & $<.0001$ \\
\hline TSH (0.34-6.0 mU/L) & 2 & $1.2-3.1$ & 1.4 & $1-2.2$ & 11,935 & $<.001$ \\
\hline INR (0.83-1.19) & 1.2 & 0.2 & 1.1 & 0.2 & -3.49 & $<.001$ \\
\hline 1,25 Hydroxy vitamin D (74.9-199.7 nmol/L) & 97.3 & $77.4-128.5$ & 97.3 & $64.9-132.3$ & 13,641 & 0.19 \\
\hline Testosterone (5.6-25.2 nmol/L) & 5.9 & $2.1-11.7$ & 5.9 & $4.4-14.6$ & 74.0 & 0.39 \\
\hline Free T3 (4.0-7.4 pmol/L) ${ }^{d}$ & 3.1 & $2.0-3.7$ & 2.3 & & & \\
\hline Free T4 (6.4-24.5 nmol/L) & 11.1 & $9.1-12.9$ & 13.1 & $11.8-16.3$ & 632.0 & 0.01 \\
\hline Prealbumin $(0.2-0.52 \mathrm{~g} / \mathrm{L})$ & 0.18 & 0.06 & 0.21 & 0.07 & 3.61 & $<.001$ \\
\hline
\end{tabular}

$A N-R$ Anorexia nervosa restricting subtype, $A N-B P$ Anorexia nervosa binge-purge subtype, $B U N$ Blood urea nitrogen, $A S T$ Aspartate aminotransferase test, $A L T$ Alanine aminotransferase test, WBC White blood cell, MCV Mean corpuscular volume, TSH Thyroid stimulating hormone, INR International normalized ratio, Free T3 Triiodothyronine, Free T4 Thyroxine

${ }^{a}$ Magnesium and TSH were available on 274 admissions. Neutrophils, lymphocytes, and 1,25 Hydroxy Vitamin D were available on 275 admissions. INR was available on 280 admissions. Testosterone was available on 19 admissions. Free T3 was available on 7 admissions. Free T4 was available on 55 admissions. Prealbumin was available on 277 admissions

${ }^{\mathrm{b}}$ Mann-Whitney $U$ test

cMedian (IQR) for not normally distributed variables

${ }^{d}$ There were too few values for an analysis between AN subtypes and Free T3 $(n=7)$

There were 179 patients (64\%) who admitted with low white blood cell counts (leukopenia), $47 \%$ with anemia and $20 \%$ with thrombocytopenia. Leukopenia and thrombocytopenia were more marked in patients with AN-R versus $A N-B P\left(M=3.810^{9} \mathrm{~L}^{-1}, \mathrm{SD}=1.6\right.$ vs. $\mathrm{M}=$ $\left.5.510^{9} \mathrm{~L}^{-1}, \mathrm{SD}=3.3, p<.0001\right)$ and $\left(\mathrm{Mdn}=19210^{9} \mathrm{~L}^{-1}\right.$, $\mathrm{IQR}=142-250$ vs. $\mathrm{Mdn}=27110^{9} \mathrm{~L}^{-1}, \mathrm{IQR}=210-387$, $p<.0001$ ), respectively (Table 3 ).

There were 60 patients on admission with a serum phosphorous level $<0.87 \mathrm{mmol} / \mathrm{L}$ and average $\% \mathrm{IBW}$ was
55.3\% ( $\mathrm{SD}=6.4)$. An additional 35\% (5 males and $93 \mathrm{fe-}$ males) developed refeeding hypophosphatemia, as early as median day $2(\mathrm{IQR}=1-2)$. Of those patients, $47 \%$ (3 males and 43 females) developed critical hypophosphatemia (serum phosphorus $<0.71 \mathrm{mmol} / \mathrm{L}$ ). $49 \%$ of patients with critical hypophosphatemia required IV phosphorus for an average of 1.6 days ( $\mathrm{SD}=0.8$ ), and all patients developing hypophosphatemia were treated for an average of 6.1 days $(\mathrm{SD}=5.1)$ of oral phosphorous repletion. Patients maintained low serum phosphorous values < 
$0.87 \mathrm{mmol} / \mathrm{L}$ for a mean of $2.1(\mathrm{SD}=1.3)$ days with use of supplement. When comparing the 98 patients who developed refeeding hypophosphatemia, with the 123 patients who never developed refeeding hypophosphatemia, there were more patients diagnosed with AN-R than patients diagnosed with AN-BP $[23 \%$ vs. $21 \%$, $\left.\chi^{2}(1)=5.44, p=.02\right]$, but there were no significant differences between gender $(p=.14)$. However, there was a significant difference in average nadir BMI between patients who developed refeeding hypophosphatemia $\left(\mathrm{M}=11.8 \mathrm{~kg} / \mathrm{m}^{2}, \mathrm{SD}=1.4\right)$ during their hospitalization compared to patients who did not $\left(\mathrm{M}=12.4 \mathrm{~kg} / \mathrm{m}^{2}, \mathrm{SD}=\right.$ 1.3, $p=.001$ ) (Table 4).

One hundred thirty-nine patients (49\%) received DXA scans during their hospitalization. Only $12 \%$ had normal BMD ( $4 \%$ male), $45 \%$ osteopenia (6\% male), and $43 \%$ osteoporosis (5\% male). The average Z-score in males was - 1.8 $(\mathrm{SD}=1.3)$ and in females, $-2.3(\mathrm{SD}=1.2)$. There were no significant differences when comparing Z-scores between patients with AN-R and AN-BP $(p=.24)$ (Table 5). The average T-score for males was $3.9(\mathrm{SD}=0.9)$, which was much worse than the average $\mathrm{T}$-score for older females $(\mathrm{M}=-2.6, \mathrm{SD}=1.0)$.

\section{Discussion}

This is the largest study ever of severely malnourished adult patients with extreme AN, with percent IBW less than $65 \%$, hospitalized on a medical stabilization unit. In comparison to our much smaller pilot study, AN patients are now more ill, manifested by an average admission BMI of $12.1 \mathrm{~kg} / \mathrm{m}^{2}$ (range $=7.5-15.7$ ) compared to our previous $13.1 \mathrm{~kg} / \mathrm{m}^{2}$ (range $=9.5-15.4$ ). Length of stay has increased from 19 to 22 days, but discharge BMI remained similar $\left(14.5 \mathrm{~kg} / \mathrm{m}^{2}\right.$ vs $\left.14.4 \mathrm{~kg} / \mathrm{m}^{2}\right)$. We have also become much more aggressive in our refeeding practices, with our admission kcals increasing from 990 to 1400 and discharge kcals increasing from $2000 \mathrm{kcal}$ to approximately $3000 \mathrm{kcal}$ [11].

When analyzed by type of eating disorder, patients with AN-R gained less weight per week than patients with AN-BP. We do not, however, believe that patients with AN-BP inherently gain weight faster than patients with AN-R, as previously suggested [12]. In that study, there was no mention of edema formation in the AN-BP group, nor how often the aldosterone antagonist spironolactone was utilized. Excessive purging behaviors lead to a secondary hyperaldosteronism (Pseudo Bartter's syndrome) which makes these patients salt and water retentive, resulting in excessive weight trends and/ or the development of edema [13, 14]. In our study, $89 \%$ of patients with AN-BP were treated with spironolactone for an average of 13 days.

Curiously, only $56 \%$ of the patients in this study were documented as having bradycardia, which is considered a universal finding in AN, especially in those patients with lower levels of body weight. Indeed, this study corroborated the correlation between bradycardia and percent IBW. Given this is a retrospective study, heart rates were obtained from the nurses' documented values, which are only obtained intermittently throughout the day; however, if 24-h telemetry values were instead used, it is likely the frequency of bradycardia would approach $100 \%$ especially with the known further lowering of heart rate at night.

Patients with AN-BP had a longer QTc interval than patients with AN-R, likely due to electrolyte imbalance. Indeed, patients with AN-BP had lower admission potassium levels which are known to prolong the QTc.

Table 4 Predictors of refeeding hypophosphatemia ${ }^{a}$

\begin{tabular}{|c|c|c|c|c|c|c|}
\hline \multirow[b]{2}{*}{ Factors } & \multicolumn{2}{|c|}{ Hypophosphatemia $(\boldsymbol{N}=98)$} & \multicolumn{2}{|c|}{ Normal Phosphorus $(\boldsymbol{N}=123)$} & \multirow[b]{2}{*}{$t$-test ${ }^{c}$} & \multirow[b]{2}{*}{$\boldsymbol{p}$ value } \\
\hline & Mean $^{\mathbf{b}}$ & $\mathrm{SD}^{\mathbf{b}}$ & Mean $^{\mathbf{b}}$ & $\mathrm{SD}^{\mathbf{b}}$ & & \\
\hline Nadir BMI & 11.8 & 1.4 & 12.4 & 1.3 & 3.31 & 0.001 \\
\hline Nadir \%IBW & 56.7 & 6.3 & 58.5 & 5.5 & 2.21 & 0.03 \\
\hline Admission Glucose (mmol/L) & 4.7 & $3.9-5.6$ & 4.4 & $3.9-5.2$ & 11,630 & 0.06 \\
\hline Admission Albumin (g/L) & 36.8 & 6.8 & 38.0 & 6.3 & -1.41 & 0.16 \\
\hline Admission Prealbumin (g/L) & 0.19 & 0.08 & 0.21 & 0.06 & -1.87 & 0.06 \\
\hline Admission WBC $\left(10^{9} \mathrm{~L}^{-1}\right)$ & 4.8 & 2.5 & 4.3 & 2.7 & -1.41 & 0.16 \\
\hline \multirow[t]{2}{*}{ Lean Mass $(\mathrm{g})^{\mathrm{d}}$} & 27,597 & 4351 & 28,578 & 5702 & -0.92 & 0.36 \\
\hline & $\mathbf{N}$ & $\%$ & $\mathbf{N}$ & $\%$ & $x^{2}$ & $p$ value \\
\hline Elevated admission AST (> $40 \mathrm{U} / \mathrm{L}$ ) & 41 & 42 & 45 & 37 & 0.63 & 0.42 \\
\hline Elevated admission ALT (> $45 \mathrm{U} / \mathrm{L}$ ) & 49 & 50 & 54 & 44 & 0.81 & 0.37 \\
\hline
\end{tabular}

$B M I$ Body mass index, IBW Ideal body weight, WBC White blood cell, AST Aspartate aminotransferase test, ALT Alanine aminotransferase test

${ }^{a}$ Hypophosphatemia defined as phosphorus $<0.87 \mathrm{mmol} / \mathrm{L}$. 60 patients with hypophosphatemia on admission were excluded

${ }^{\mathrm{b}}$ Median (IQR) for not normally distributed variables

"Mann-Whitney $U$ test

${ }^{\mathrm{d}}$ Data for lean mass (g) was available for 38 patients with refeeding hypophosphatemia and 66 patients with normal phosphorus 
Table 5 Bone mineral density and lipodystrophy studies between AN subtypes

\begin{tabular}{|c|c|c|c|c|c|c|c|}
\hline \multirow[b]{2}{*}{ DXA scans } & \multirow[t]{2}{*}{ Cohort $(\boldsymbol{N}=139)$} & \multicolumn{2}{|l|}{$\begin{array}{l}\text { AN-R } \\
(\boldsymbol{N}=94)\end{array}$} & \multicolumn{2}{|l|}{$\begin{array}{l}\text { AN-BP } \\
(\boldsymbol{N}=45)\end{array}$} & & \\
\hline & & $\mathrm{N}$ & $\%$ & $\mathrm{~N}$ & $\%$ & & \\
\hline Normal BMD (Z/T-score > -1) & 17 & 11 & 65 & 6 & 35 & & \\
\hline Osteopenia (Z/T-score -1 to -2.5$)$ & 62 & 45 & 73 & 17 & 27 & & \\
\hline \multirow[t]{2}{*}{ Osteoporosis (Z/T-score < - 2.5) } & 60 & 38 & 63 & 22 & 37 & & \\
\hline & & Mean & SD & Mean & SD & $t$-test & $p$ value \\
\hline Z-score & 127 & -2.2 & 1.2 & -2.4 & 1.1 & -1.17 & 0.24 \\
\hline T-score ${ }^{a}$ & 12 & -2.7 & 1.2 & -3.7 & 0.4 & & \\
\hline \multicolumn{8}{|l|}{ Lipodystrophy studies } \\
\hline & & Median & IQR & Median & IQR & $\begin{array}{l}\text { Mann-Whitney } \\
U \text { test }\end{array}$ & $p$ value \\
\hline Body Fat (g) & 134 & 3799 & $3398-4709$ & 4329 & $3566-5317$ & 3408 & 0.04 \\
\hline Body Fat (\%) & 134 & 11.6 & $10.8-13.4$ & 12.8 & $11.2-16.1$ & 3486 & 0.01 \\
\hline Lean Mass (\%) & 134 & 77.8 & $75.2-80.0$ & 76.4 & $74.4-78.1$ & 2465 & 0.02 \\
\hline
\end{tabular}

$A N-R$ Anorexia nervosa restricting subtype, $A N-B P$ Anorexia nervosa binge-purge subtype, $D X A$ Dual-energy $\mathrm{X}$-ray absorptiometry, $B M D$ Bone mineral density, IQR Interquartile range

${ }^{a}$ There were too few values for an analysis between AN subtypes and T-scores $(n=12)$

Importantly, once again, no correlation was found between QTc and admission BMI thus reinforcing the fact that a prolonged QTc is not inherent to AN. Rather, when detected, a prolonged QTc is a consequence of electrolyte disturbances or the usage of QT-prolonging medications [15-17].

In our cohort, $70 \%$ of patients had abnormal LFTs which peaked on the fourth hospital day for patients with AN-R and day five for patients with AN-BP. On average, the ALT was greater than the AST across all subtypes of eating disorders with AN-R patients having higher transaminases than AN-BP patients. Elevations in transaminases are the most common kind of liver abnormality seen in patients with severe AN [18, 19]; bilirubin and alkaline phosphatase levels are almost never elevated. In our cohort, $40 \%$ of patients with a BMI $<12.1$ $\mathrm{kg} / \mathrm{m}^{2}$ had elevated transaminases, thus supporting the relationship between severity of AN and likelihood of transaminitis. The most likely pathophysiologic mechanism is starvation-mediated autophagy [20,21].

Hypoglycemia was more likely to develop in those with elevation of transaminases greater than three times nor$\mathrm{mal}$ and in those with greater severity of malnutrition, as evidenced by $27 \%$ of those with AN-R developing hypoglycemia vs. $11 \%$ of individuals with AN-BP. Thus, hypoglycemia as a surrogate of severity of illness was supported $[22,23]$. This is very important because our experience suggests lean people do not typically have the customary adrenergic or neuroglycopenic warning symptoms typical of severe hypoglycemia [22].

Serum albumin levels were notably within the normal range, notwithstanding their marked malnutrition. However, more than $50 \%$ of our patients admitted with a low prealbumin level $<0.2 \mathrm{~g} / \mathrm{L}$, with $A N-\mathrm{R}$ patients having the lowest values. These findings are supported by an emerging literature that albumin is not considered a reliable indicator of the severity of AN [23, 24]. Rather, albumin is best viewed as an acute phase reactant that decreases predictably in critical illness such as sepsis [25]. The precise cause of low serum prealbumin levels in severe AN remains to be elucidated $[24,26]$. Furthermore, low serum prealbumin is associated with both refeeding hypophosphatemia [27] as well as hypoglycemia in AN.

Patients with AN-R had more pronounced leukopenia and thrombocytopenia than those with AN-BP. In severe $\mathrm{AN}$, the bone marrow shows signs of serous fat atrophy [28-30], which is directly related to duration of illness and BMI [31]. This corrects with a judicious nutritional plan.

The most common electrolyte abnormality on admission, in our cohort, was hypokalemia, with $33 \%$ of patients having a serum potassium level $<3.6 \mathrm{mmol} /$ $\mathrm{L}$, followed by hyponatremia. Hypernatremia was very rare. When examined by eating disorder sub-type, patients with AN-BP had, not surprisingly, statistically significant lower sodium and potassium levels on admission. The hyperaldosteronism resulting from the dehydration of purging along with loss of potassium and other electrolytes in emesis, stool, and urine contribute to hypokalemia [32, 33]. The ability to correct hypokalemia is improved with down-regulation of aldosterone secretion, which can be accomplished with intravascular volume repletion; hence the large number of patients that received IV fluids in the treatment of their hypokalemia. 
Notably, only $21 \%$ of this cohort admitted with hypophosphatemia, but over one third of our patients subsequently developed refeeding hypophosphatemia by hospital day two. Compared to patients who did not develop hypophosphatemia, those who did, had lower admission BMI, thus representing a sicker patient population who is at greater risk for refeeding hypophosphatemia [34]. Studies have indeed shown that as BMI nears $12 \mathrm{~kg} / \mathrm{m}^{2}$, medical complications such as refeeding hypophosphatemia occur more frequently $[27,35,36]$. Based on this present study, patients who manifested a tendency towards developing refeeding hypophosphatemia had lower BMIs, higher transaminases, and lower serum prealbumin levels. Caloric intake during admission, per se, did not predict hypophosphatemia. However, notwithstanding the extremely ill population of patients cared for on ACUTE, 44\% never developed refeeding hypophosphatemia. We therefore feel it unnecessary to prophylactically give phosphorus replacement to all patients at the time of admission given the possibility that it can cause diarrhea.

Lastly, 139 of our patients received a DXA scan during their admission and of those, $88 \%$ were found to have BMD loss [osteopenia (45\%) and osteoporosis $(43 \%)]$. Although prior studies have shown rates of osteoporosis in males to be worse than females [37], in our current study, females had overall higher rates of osteoporosis ( $43 \%$ vs $5 \%$ ). Weight restoration is the gold standard for treatment of osteoporosis [38, 39]. Further studies are needed to elucidate which available pharmaceutical options may also be valuable for this patient population [40].

While the strength of this article is that it describes the medical complications in the largest ever published cohort of male and female patients with extreme AN, there are limitations. First, it was based on retrospective data review, thereby somewhat limiting the conclusions that can be drawn from this descriptive study. Secondly, we included patients transferred from other hospitals as well as other eating disorder treatment facilities and we cannot account for the care and refeeding protocols the patients received there. Third, our sample size remains relatively small as our program focuses exclusively on the care of the extreme form of AN and may reduce the generalizability of our findings. Lastly, we did not include readmissions in this sample size.

In conclusion, this article methodically examines the many medical complications of patients with extreme AN-R and AN-BP in an effort to inform care providers of the multitude of medical issues which may be present in these patients with extreme forms of the illness.

\section{Abbreviations}

ACUTE: ACUTE Center for Eating Disorders at Denver Health; ALT: Alanine aminotransferase; AMA: Against medical advice; AN: Anorexia nervosa; AN-
BP: Binge-purge subtype; AN-R: Restricting subtype; AST: Aspartate aminotransferase; BMD: Bone mineral density; BMl: Body mass index; BPM: Beats per minute; DSM: Diagnostic and Statistical Manual of Mental Disorders; DXA: Dual-energy X-ray absorptiometry; EKG: Electrocardiogram; IBW: Ideal body weight; ICU: Intensive care unit; IQR: Interquartile range; IV: Intravenous; LFT: Liver function test; M: Mean; MDN: Median; MHH: Mental health hold; PHP: Partial hospitalization program; SAS: Statistical analysis software; SD: Standard deviation; SIADH: Syndrome of inappropriate antidiuretic hormone; STC: Short-term certification

\section{Acknowledgements \\ Not applicable.}

\section{Authors' contributions}

DG interpreted data and was a major contributor to writing the manuscript. AW compiled and analyzed the data and was a major contributor to writing the manuscript. JC and MM were major contributors in writing the manuscript and in interpreting outcomes. PM conceptualized the study and contributed to writing of the manuscript. All authors read and approved the final manuscript.

Funding

This study was unfunded.

\section{Availability of data and materials}

The datasets generated and analyzed during the current study are available from the corresponding author on reasonable request.

\section{Ethics approval and consent to participate}

This study was reviewed and approved by the Colorado Multiple Institutional Review Board (Protocol \# 17-7808). The Board determined that the retrospective study met criteria for a full waiver of consent and for a full waiver of HIPAA authorization

\section{Consent for publication}

Not applicable.

\section{Competing interests}

PM and JC report equity in the Eating Recovery Center-Denver. DG, AW and MM declare that they have no competing interests.

\section{Author details}

${ }^{1}$ ACUTE Center for Eating Disorders at Denver Health, 723 Delaware St, Pav M 3rd floor, Denver, CO 80204, USA. ${ }^{2}$ Department of Medicine, University of Colorado School of Medicine, 13001 E 17th PI, Aurora, CO 80045, USA.

${ }^{3}$ Eating Recovery Center, 7351 E Lowry Blvd, Denver, CO 80230, USA.

Received: 16 January 2020 Accepted: 21 May 2020

Published online: 23 June 2020

\section{References}

1. Mehler PS, Brown CA. Anorexia nervosa - medical complications. J Eat Disord. 2015:3:11.

2. Fichter MM, Quadflieg N. Mortality in eating disorders - results of a large prospective clinical longitudinal study. Int J Eat Disord. 2016;49(4):391-401.

3. Franko DL, Keshaviah A, Eddy KT, Krishna M, Davis MC, Keel PK, et al. A longitudinal investigation of mortality in anorexia nervosa and bulimia nervosa. Am J Psychiatry. 2013;170(8):917-25.

4. Pompili M, Mancinelli I, Girardi P, Ruberto A, Tatarelli R. Suicide in anorexia nervosa: a meta-analysis. Int J Eat Disord. 2004;36(1):99-103.

5. Eddy KT, Tabri N, Thomas JJ, Murray HB, Keshaviah A, Hastings E, et al. Recovery from anorexia nervosa and bulimia nervosa at 22-year follow-up. J Clin Psychiatry. 2017;78(2):184-9.

6. Baran SA, Weltzin TE, Kaye WH. Low discharge weight and outcome in anorexia nervosa. Am J Psychiatry. 1995;152(7):1070-2.

7. Rigaud D, Pennacchio H, Bizeul C, Reveillard V, Verges B. Outcome in AN adult patients: a 13-year follow-up in 484 patients. Diabetes Metab. 2011; 37(4):305-11.

8. Misra M, Aggarwal A, K Miller K, Almazan C, Worley M, Soyka L, et al. Effects of anorexia nervosa on clinical, hematologic, biochemical, and bone density 
parameters in community-dwelling adolescent girls. Pediatrics. 2005;114 1574-83.

9. APA. Diagnostic and Statistical Manual of Mental Disorders. 5th ed. Washington: APA; 2013.

10. Hamwi GJ. Therapy: Changing dietary concepts. In: S Danowski T, editor. Diabetes mellitus: Diagnosis and treatment. New York: American Diabetes Association; 1964. p. 73-8.

11. Gaudiani JL, Sabel AL, Mascolo M, Mehler PS. Severe anorexia nervosa: outcomes from a medical stabilization unit. Int J Eat Disord. 2012;45(1):85-92.

12. Makhzoumi SH, Coughlin JW, Schreyer CC, Redgrave GW, Pitts SC, Guarda AS. Weight gain trajectories in hospital-based treatment of anorexia nervosa. Int J Eat Disord. 2017:50(3):266-74.

13. Bahia A, Mascolo M, Gaudiani JL, Mehler PS. PseudoBartter syndrome in eating disorders. Int J Eat Disord. 2012;45(1):150-3.

14. Mascolo M, Chu ES, Mehler PS. Abuse and clinical value of diuretics in eating disorders therapeutic applications. Int J Eat Disord. 2011;44(3):200-2.

15. Krantz MJ, Sabel AL, Sagar U, Long CS, Barbey JT, White KV, et al. Factors influencing QT prolongation in patients hospitalized with severe anorexia nervosa. Gen Hosp Psychiatry. 2011;34(2):173-7.

16. Facchini M, Sala L, Malfatto G, Bragato R, Redaelli G, Invitti C. Low-K+ dependent QT prolongation and risk for ventricular arrhythmia in anorexia nervosa. Int J Cardiol. 2006;106(2):170-6.

17. Mehler PS, Blalock DV, Walden K, Kaur S, McBride J, Walsh K, et al. Medical findings in 1,026 consecutive adult inpatient-residential eating disordered patients. Int J Eat Disord. 2018;51(4):305-13.

18. Rosen E, Sabel AL, Brinton JT, Catanach B, Gaudiani JL, Mehler PS. Liver dysfunction in patients with severe anorexia nervosa. Int J Eat Disord. 2016; 49(2):151-8

19. Ozawa $Y$, Shimizu T, Shishiba Y. Elevation of serum aminotransferase as a sign of multiorgan-disorders in severely emaciated anorexia nervosa. Internal medicine (Tokyo, Japan). 1998;37(1):32-9.

20. Rautou PE, Cazals-Hatem D, Moreau R, Francoz C, Feldmann G, Lebrec D, et al. Acute liver cell damage in patients with anorexia nervosa: a possible role of starvation-induced hepatocyte autophagy. Gastroenterology. 2008; 135(3):840-8 8 e1-3.

21. Kheloufi M, Boulanger CM, Durand F, Rautou PE. Liver autophagy in anorexia nervosa and acute liver injury. Biomed Res Int. 2014;2014:701064.

22. Wexler DJ, Macias-Konstantopoulos W, Forcione DG, Xiong L, Cauley CE, Pierce KJ. Case 23-2018: a 36-year-old man with episodes of confusion and hypoglycemia. N Engl J Med. 2018;379(4):376-85.

23. Narayanan V, Gaudiani JL, Mehler PS. Serum albumin levels may not correlate with weight status in severe anorexia nervosa. Eat Disord. 2009; 17(4):322-6.

24. Huysentruyt K, De Schepper J, Vanbesien J, Vandenplas Y. Albumin and prealbumin levels do not reflect the nutritional status of female adolescents with restrictive eating disorders. Acta Paediatr. 2016;105(4):e167-9.

25. Fuhrman MP, Charney P, Mueller CM. Hepatic proteins and nutrition assessment. J Am Diet Assoc. 2004;104(8):1258-64.

26. Gaudiani JL, Sabel AL, Mehler PS. Low prealbumin is a significant predictor of medical complications in severe anorexia nervosa. Int J Eat Disord. 2014 47(2):148-56.

27. Brown CA, Sabel AL, Gaudiani JL, Mehler PS. Predictors of hypophosphatemia during refeeding of patients with severe anorexia nervosa. Int J Eat Disord. 2015:48(7):898-904

28. Cleary BS, Gaudiani JL, Mehler PS. Interpreting the complete blood count in anorexia nervosa. Eat Disord. 2010;18(2):132-9.

29. Mohamed M, Khalafallah A. Gelatinous transformation of bone marrow in a patient with severe anorexia nervosa. Int J Hematol. 2013;97(2):157-8.

30. Hutter G, Ganepola S, Hofmann WK. The hematology of anorexia nervosa. Int J Eat Disord. 2009;42(4):293-300

31. De Filippo E, Marra M, Alfinito F, Di Guglielmo ML, Majorano P, Cerciello G, et al. Hematological complications in anorexia nervosa. Eur J Clin Nutr. 2016:70(11):1305-8.

32. Imbierowicz K, Curkovic I, Braks K, Geiser F, Liedtke R, Jacoby GE. Effect of weight-regulating practices on potassium level in patients with anorexia or bulimia nervosa. Eur Eat Disord Rev. 2004;12(5):300-6.

33. Mascolo M, McBride J, Mehler PS. Effective medical treatment strategies to help cessation of purging behaviors. Int J Eat Disord. 2016;49(3):324-30.

34. National Institute for Health and Clinical Excellence. NICE quick reference guide: nutrition support in adults: oral nutrition support, enteral tube feeding and parenteral nutrition clinical guideline 32. London: National Institute for Health and Clinical Excellence; 2006.

35. Sachs KV, Andersen DM, Sommer J, Winkelman AB, Mehler PS. Avoiding medical complications during the refeeding of patients with anorexia nervosa. Eat Disord. 2015;23(5):411-21.

36. O'Connor G, Nicholls D. Refeeding hypophosphatemia in adolescents with anorexia nervosa: a systematic review. Nutr Clin Pract. 2013;28(3):358-64.

37. Mehler PS, Sabel AL, Watson TL, Andersen AE. High risk of osteoporosis in male patients with eating disorders. Int J Eat Disord. 2008;41(7):666-72.

38. El Ghoch M, Gatti D, Calugi S, Viapiana O, Bazzani PV, Dalle GR. The Association between Weight Gain/Restoration and Bone Mineral Density in Adolescents with Anorexia Nervosa: A Systematic Review. Nutrients. 2016; 8(12):769

39. Misra M, Prabhakaran R, Miller KK, Goldstein MA, Mickley D, Clauss L, et al. Weight gain and restoration of menses as predictors of bone mineral density change in adolescent girls with anorexia nervosa-1. J Clin Endocrinol Metab. 2008:93(4):1231-7.

40. Drabkin A, Rothman MS, Wassenaar E, Mascolo M, Mehler PS. Assessment and clinical management of bone disease in adults with eating disorders: a review. J Eat Disord. 2017:5:42.

\section{Publisher's Note}

Springer Nature remains neutral with regard to jurisdictional claims in published maps and institutional affiliations.
Ready to submit your research? Choose BMC and benefit from:

- fast, convenient online submission

- thorough peer review by experienced researchers in your field

- rapid publication on acceptance

- support for research data, including large and complex data types

- gold Open Access which fosters wider collaboration and increased citations

- maximum visibility for your research: over $100 \mathrm{M}$ website views per year

At $\mathrm{BMC}$, research is always in progress.

Learn more biomedcentral.com/submissions 